PROCEEDINGS OF THE AMERICAN MATHEMATICAL SOCIETY

Volume 124, Number 6, June 1996

\title{
DISCRETE VALUATION OVERRINGS OF NOETHERIAN DOMAINS
}

\author{
PAUL-JEAN CAHEN, EVAN G. HOUSTON, AND THOMAS G. LUCAS \\ (Communicated by Wolmer V. Vasconcelos)
}

\begin{abstract}
We show that, given a chain $0=P_{0} \subset P_{1} \subset \cdots \subset P_{n}$ of prime ideals in a Noetherian domain $R$, there exist a finitely generated overring $T$ of $R$ and a saturated chain of primes in $T$ contracting term by term to the given chain. We further show that there is a discrete rank $n$ valuation overring of $R$ whose primes contract to those of the given chain.
\end{abstract}

Let $R$ be an integral domain (with 1). It is well known that if $0=P_{0} \subset P_{1} \subset$ $\cdots \subset P_{n}$ is a chain of prime ideals in $R$, then there exist a valuation overring $V$ of $R$ and a chain of primes $0=Q_{0} \subset Q_{1} \subset \cdots \subset Q_{n}$ in $V$ with $Q_{i} \cap R=P_{i}$ for $i=1, \ldots, n$. On the other hand, Chevalley showed [C] that if $R$ is Noetherian and $P$ is any nonzero prime of $R$, then there is a discrete rank 1 valuation overring of $R$ centered on $P$. D. D. Anderson has asked whether these two results can be "combined": given a Noetherian domain $R$ and a chain as above, is there a discrete rank $n$ valuation overring of $R$ whose primes contract to those of the given chain? (Recall that a finite-dimensional valuation ring $V$ is discrete if $P V_{P}$ is principal for each prime ideal $P$ of $V$.)

We show that this question has an affirmative answer. In fact, we prove the following stronger result.

Theorem. Let $0=P_{0} \subset P_{1} \subset \cdots \subset P_{n}$ be a chain of primes in the Noetherian domain $R$, and let $s_{1}, \ldots, s_{n}$ be a sequence of integers with $1 \leq s_{i} \leq \operatorname{ht}\left(P_{i} / P_{i-1}\right)$. Then there exist an overring $T$ of $R$ and a chain of primes $0=Q_{0} \subset Q_{1} \subset \cdots \subset Q_{n}$ in $T$ such that $Q_{i} \cap R=P_{i}$ and $\operatorname{ht}\left(Q_{i} / Q_{i-1}\right)=s_{i}$ for $i=1, \ldots, n$. Moreover, $T$ can be taken to be either a finitely generated extension of $R$ or a discrete valuation ring of rank $s=\sum s_{i}$.

We use " $\subset$ " to denote proper containment. For a prime ideal $P$ of a (commutative) ring $R$, an upper to $P$ is a prime ideal $U$ of $R[X]$ for which $U \cap R=P$ and $U \neq P[X]$. An overring of a domain is understood to have the same quotient field as the base domain. Other terminology is standard as in [G].

Lemma 1. Let $P$ be a prime ideal in a Noetherian ring $R$, and let $a, b \in R \backslash P$. If there is no prime $p$ for which $P \subset p$, ht $(p / P)=1$, and $a, b \in p$, then $\operatorname{rad}(P, a X-b) R[X]$ is an upper to $P$ in $R[X]$.

Proof. Let $U$ be a prime of $R[X]$ minimal over $(P, a X-b) R[X]$. By the principal ideal theorem, $\operatorname{ht}(U / P R[X])=1$. Hence either $U$ is an upper to $P$ or $U=p[X]$,

Received by the editors October 24, 1994 and, in revised form, December 16, 1994

1991 Mathematics Subject Classification. Primary 13E05, 13A18; Secondary 13G05, 13A15.

(C)1996 American Mathematical Society 
where $p=U \cap R$. In the latter case, we have $P \subset p, \operatorname{ht}(p / P)=1$, and $a, b \in p$, contradicting the hypothesis. Thus any prime minimal over $(P, a X-b) R[X]$ is an upper to $P$, and since $a X-b$ has degree 1 , only one upper to $P$ can contain it. That upper is clearly $\operatorname{rad}(P, a X-b) R[X]$.

We now prove the first case of our theorem; that is, we construct a finitely generated overring $T$ of $R$ such that $T$ contains the indicated chain of primes. Our proof may be regarded as a refinement of the proof of Chevalley's result presented by Kaplansky in [K2].

Proof. Choose an $r$ with $1 \leq r \leq n$ for which $\operatorname{ht}\left(P_{r} / P_{r-1}\right) \geq 2$. We will produce a simple overring $R[u]$ of $R$ and a chain of primes $0=Q_{0} \subset Q_{1} \subset \cdots \subset Q_{r-1} \subset Q_{r} \subset$ $\cdots \subset Q_{n}$ in $R[u]$ such that $Q_{i} \cap R=P_{i}$ for $0 \leq i \leq n, \operatorname{ht}\left(Q_{i} / Q_{i-1}\right)=\operatorname{ht}\left(P_{i} / P_{i-1}\right)$ for $i \neq r$, and $\operatorname{ht}\left(Q_{r} / Q_{r-1}\right)=\operatorname{ht}\left(P_{r} / P_{r-1}\right)-1$. The first case of the theorem will follow from an iteration of this process.

Pick $a \in P_{r} \backslash P_{r-1}$ and, for $0 \leq i \leq r-1$, let $S_{i}=\left\{p \in \operatorname{Spec}(R) \mid P_{i} \subset p\right.$, $a \in p$, and ht $\left.\left(p / P_{i}\right)=1\right\}$. Note that each $S_{i}$ is finite. Also, since ht $\left(P_{r} / P_{i}\right) \geq 2$, $P_{r} \nsubseteq \nsubseteq p$ for $p \in S_{i}$. By prime avoidance, we may choose $b \in P_{r} \backslash \bigcup S_{i}$. By Lemma 1 , $U_{i}=\operatorname{rad}(P, a X-b) R[X]$ is an upper to $P_{i}$ for $0 \leq i \leq r-1$. Since $a, b \in P_{r}$, we see that $U_{r-1} \subset P_{r}[X]$, and we have the following chain of primes in $R[X] / U_{0}$ :

$$
U_{0} / U_{0} \subset U_{1} / U_{0} \subset \cdots \subset U_{r-1} / U_{0} \subset P_{r}[X] / U_{0} \subset P_{r+1}[X] / U_{0} \subset \cdots \subset P_{n}[X] / U_{0} .
$$

Since $U_{0}$ is an upper to zero containing $a X-b, R[X] / U_{0}$ can be identified with the simple overring $R[u]$, where $u=b / a$. We claim that the chain (in $R[u]$ which identifies with the chain) above satisfies our requirements. It is clear that it contracts to the original chain in $R$. Therefore, to complete the argument, it suffices to show that $\operatorname{ht}\left(U_{i} / U_{i-1}\right)=\operatorname{ht}\left(P_{i} / P_{i-1}\right)$ for $1 \leq i \leq r-1$, that $\operatorname{ht}\left(P_{r}[X] / U_{r-1}\right)=\operatorname{ht}\left(P_{r} / P_{r-1}\right)-1$, and that $\operatorname{ht}\left(P_{i}[X] / P_{i-1}[X]\right)=\operatorname{ht}\left(P_{i} / P_{i-1}\right)$ for $r+1 \leq i \leq n$. The last of these three statements is clear, and the first two are easy exercises using the generalized principal ideal theorem [K1, Theorem 154] (using the fact that $U_{i} / P_{i}[X]$ is the radical of a principal ideal in the ring $\left.R[X] / P_{i}[X] \simeq\left(R / P_{i}\right)[X]\right)$. This completes the argument for the first case of our theorem.

The second case of the theorem will be proved in two steps. The next lemma covers the situation where the given chain $0=P_{0} \subset P_{1} \subset \cdots \subset P_{n}$ is saturated in $R$.

Lemma 2. Let $R$ be a Noetherian domain with quotient field $K$, let $0=P_{0} \subset P_{1} \subset$ $\cdots \subset P_{n}$ be a saturated chain of primes in $R$, and let $L$ be a finite extension field of $K$. Then there exists a discrete rank $n$ valuation domain $V$ with quotient field $L$ such that, if $0=Q_{0} \subset Q_{1} \subset \cdots \subset Q_{n}$ are the primes of $V$, then $Q_{i} \cap R=P_{i}$ and the quotient field of $V / Q_{i}$ is a finite extension of the quotient field of $R / P_{i}$ for each $i=1, \ldots, n$.

Proof. First, assume $n=1$. Since localizing at $P_{1}$ does not change the quotient field of $R / P_{1}$, we may assume (for the case under consideration) that $R$ is local with maximal ideal $P_{1}$ (and $\operatorname{dim}(R)=1$ ). By [N, 33.10], the integral closure $R^{\prime}$ of $R$ is a Krull domain, and $R_{Q}^{\prime} / Q R_{Q}^{\prime} \simeq R^{\prime} / Q$ is a finite extension of $R / P_{1}$, where $Q$ is a maximal ideal of $R^{\prime}$. Of course, $R_{Q}^{\prime}$ is a discrete rank 1 valuation domain. Since $L$ is a finite extension of $K$, there is a discrete rank 1 valuation domain 
$\left(V, Q_{1}\right)$ with quotient field $L$ such that $V \cap K=R_{Q}^{\prime}$ and $Q_{1} \cap R^{\prime}=Q R_{Q}^{\prime}[\mathrm{G}$, Section 19] and such that $V / Q_{1}$ is a finite extension of $R_{Q}^{\prime} / Q R_{Q}^{\prime}$ (and hence also of $R / P_{1}$ ) [G, Lemma 41.4]. This takes care of the case $n=1$. Inductively, assume that $W$ is a discrete rank $n-1$ valuation ring with quotient field $L$ such that, if $0=Q_{0} \subset \cdots \subset Q_{n-1}$ are the primes of $W$, then $Q_{i} \cap R=P_{i}$ and the quotient field of $W / Q_{i}$ is a finite extension of that of $R / P_{i}$ for each $i=1, \ldots, n-1$. In particular, $W / Q_{n-1}$ is a finite extension of the quotient field of $R / P_{n-1}$. Then since $P_{n} / P_{n-1}$ is a height one prime of the Noetherian domain $R / P_{n-1}$, by the case $n=1$, there is a discrete rank 1 valuation ring $\left(\bar{V}, \bar{Q}_{n}\right)$ with quotient field $W / Q_{n-1}$ such that $\bar{Q}_{n} \cap\left(R / P_{n-1}\right)=P_{n} / P_{n-1}$ and such that $\bar{V} / \bar{Q}_{n}$ is a finite extension of the quotient field of $R / P_{n-1}$. Let $V$ be the inverse image of $\bar{V}$ under the natural map $W \rightarrow W / Q_{n-1}$. It is not difficult to verify that this $V$ does what is required.

We now prove the second case of our theorem. That is, we construct a discrete valuation overring $T$ of $R$ and an appropriate chain of primes in $T$.

Proof. By the first case of the theorem, there exist a finitely generated overring $S$ of $R$ and a chain of primes $0=Q_{0} \subset Q_{1} \subset \cdots \subset Q_{n}$ in $S$ such that $Q_{i} \cap R=P_{i}$ and $\operatorname{ht}\left(Q_{i} / Q_{i-1}\right)=s_{i}$ for $i=1, \ldots, n$. For each $i$, insert between $Q_{i-1}$ and $Q_{i}$ a subchain of length $s_{i}$; this produces a saturated chain of primes of length $s=\sum s_{i}$ from 0 to $Q_{n}$ in $S$. Now apply Lemma 2 to obtain the required $T$.

\section{ACKNOWLEDGMENT}

The authors express gratitude to the referee for sharpening both the statement and the proof of our theorem.

\section{REFERENCES}

[C] C. Chevalley, La notion d'anneau de décomposition, Nagoya Math. J., 7 (1954), 21-33. MR 16:788g

[G] R. Gilmer, Multiplicative ideal theory, Dekker, New York, 1972. MR 55:323

[K1] I. Kaplansky, Commutative rings, Allyn and Bacon, Chicago, 1970. MR 40:7234

[K2] I. Kaplansky, Topics in commutative ring theory, University of Chicago lecture notes (1974). MR 55:322

[N] M. Nagata, Local rings, Krieger, New York, 1975. MR 57:301

[SL] A. de Souza Doering and Y. Lequain, Chains of prime ideals in polynomial rings, J. Algebra, 78 (1982), 163-180. MR 84d:13008

(P.-J. Cahen) Service de Mathématiques 322, Faculté des Sciences de SaintJÉrôme, 13397 Marseille CEDEx 20, CNRS URA 225, France

E-mail address: cahen@vmesal2.u-3mrs.fr

(E. G. Houston and T. G. Lucas) Department of Mathematics, University of North Carolina at Charlotte, Charlotte, North Carolina 28223

E-mail address, E. G. Houston: fma00egh@unccvm.uncc.edu

E-mail address, T. G. Lucas: tglucas@unccsun.uncc.edu 